\title{
Graphene Coated Scaffold for Bone Tissue \\ Engineering: Physicochemical and Osteogenic \\ Characterizations
}

\section{Sajad Bahrami}

Department of Orthopedic Surgery, AJA University of Medical Sciences, Tehran

Nafiseh Baheiraei ( $\sim$ n.baheiraei@modares.ac.ir)

Department of Anatomical science, Faculty of Medical Sciences, Tarbiat Modares University, Tehran

Mostafa Shahrezaee

Department of Orthopedic Surgery, AJA University of Medical Sciences, Tehran

\section{Research Article}

Keywords: Tissue engineering, nanotechnology, collagen, graphene, Bone

Posted Date: April 15th, 2021

DOl: https://doi.org/10.21203/rs.3.rs-408730/v1

License: (c) (1) This work is licensed under a Creative Commons Attribution 4.0 International License.

Read Full License

Version of Record: A version of this preprint was published at Scientific Reports on August 18th, 2021. See the published version at https://doi.org/10.1038/s41598-021-96271-1. 


\section{Abstract}

Variety of bone-related diseases and injures and limitations of traditional regeneration methods need to introduce new tissue substitutes. Tissue engineering and regeneration combined with nanomedicine can provide different natural or synthetic and combined scaffolds with bone mimicking properties for implant in the injured area. In this study, we synthesized collagen (Col) and reduced graphene oxide coated collagen (Col-rGO) scaffolds and evaluated their in vitro and in vivo effects on bone tissue repair. Col and Col-rGO scaffolds were synthesized by chemical crosslinking and freeze-drying methods. The surface topography, mechanical and chemical properties of scaffolds were characterized and showed threedimensional (3D) porous scaffolds and successful coating of rGO on Col. rGO coating enhanced mechanical strength of Col-rGO scaffolds compared with Col scaffolds by 2.8 folds. Furthermore, Col-rGO scaffolds confirmed that graphene addition not only did not any cytotoxic effects but also enhanced human bone marrow-derived mesenchymal stem cells (hBMSCs) viability and proliferation with 3D adherence and expansion. Finally, scaffolds implantation into rabbit cranial bone defect for 12 weeks showed increased bone formation, confirmed by Hematoxylin-Eosin (H\&E) and alizarin red staining. Altogether, the study showed that rGO coating improves Col scaffold properties and could be a promising implant for bone injuries.

\section{Introduction}

Bone tissue has a remarkable capability of self-healing in small defects. However, larger defects result from osteoporosis, tumors, traumatic fractures and infections cannot regenerate spontaneously, resulting fractures and deformities ${ }^{1}$. Traditionally, allografts and autografts are used as the common treatment method for bone defects for about 2.2 million grafts annually worldwide. Autografts are the most prevalent treatment method, but they are limited by donor site morbidity, high failure rates (up to $50 \%$ ) and limited graft supply ${ }^{1,2}$. Also, metallic fixtures that are used concurrently with grafts for bone fragments stabilization cause discomfort, infections, long-term complications and repeated surgeries in patients, especially in children ${ }^{1}$. In recent years, new methods have been introduced for fabrication of less problematic bone tissue grafts. In this regard, bone tissue engineering possesses great potential for regeneration purposes.

Tissue engineering introduces structural and functional alternatives for human tissue imitation called the scaffold, natural and/or synthetic materials for implant into tissue defects. Scaffolds can imitate the natural three-dimensional (3D) structure of human tissue better than traditional cell culture twodimensional (2D) plates. Scaffolds are 3D porous structures with similar physicochemical properties with extracellular matrix (ECM) of the target tissue. In general, scaffolds are biocompatible and biodegradables and provide appropriate physical, mechanical and biochemical support for cell growth and differentiation ${ }^{3,4}$. Optimal bone tissue engineering scaffold presents different structural, biological and physicochemical characteristics for bone tissue. Bone tissue scaffolds should have 3D and porous structures for in vivo like cell growth and connections and oxygen/nutrients diffusion. Appropriate 
biocompatibility and biodegradability provide additional properties for biological compatibility. Mechanical strength, osteoinductivity and osteoconductivity properties could also be useful for more relevant results and practical clinical applications ${ }^{5,67}$.

In human tissue, cells surround by microenvironment including ECM and other cells. Microenvironment applies a wide range of stimuli from macro- to nanoscales, chemical, mechanical and biologic factors that affect on cell/ tissue fate ${ }^{7,8}$. Bone ECM includes $30 \%$ collagen (Col) fibrils and $70 \%$ calcium phosphate crystals. Col is the most abundant protein in body tissue and is widely used in biomaterials applications. Col exists in the bone matrix with four different types in which type I forms $97 \%$ of Col part 1,6. Col type I is a heterotrimer with about 1000 amino acids and $300 \mathrm{~nm}$ length ${ }^{9}$. Col scaffolds have widely used for bone tissue engineering due to abundance, biocompatibility, biodegradability, high porosity, hydrophilicity and low antigenicity ${ }^{6,9}$. For example, Sheyn, D. et al. ${ }^{10}$ used biodegradable Col scaffold cultured with mesenchymal stem cells to repair radius bone defects. $\mu \mathrm{CT}$ results showed complete bone repair after eight weeks. High bone density also showed in addition with increased osteogenic markers, BSP and OC. Arakawa et al. fabricated photocrosslinkable methacrylated glycol chitosan (MeGC) and Col hydrogel and showed enhanced bone marrow-derived mesenchymal stem cells (BMMSCs) attachment, spreading, proliferation and osteogenic differentiation ${ }^{11}$.

On the other side, natural materials encounter limitations, especially for in vivo applications, mostly physicochemical limitations. Novel tissue engineering strategies could overcome these limitations. Bone tissue scaffolds need to have sufficient mechanical properties. Col suffers weak mechanical properties, causing limited applications ${ }^{6,9}$. Different Col composite with bioceramic components like hydroxyapatite, polymer components like silk fibroin and nanomaterials have been used in bone tissue engineering studies ${ }^{9}$. Combination of Col with synthetic materials could help strength enhancement and fabrication of in vivo imitating bone scaffold. For example, in our previous study, Col/ $\beta$-tricalcium phosphate bone graft improved the Col scaffolds' mechanical and biological properties and differentiation of BMMSCs into osteoblasts ${ }^{12}$. It is investigated that Bioglass $45 S 5$ (BG) incorporated methacrylated Col 3D printed constructs allowed improved stability, reduced swelling of Col hydrogels, enhanced alkaline phosphatase activity of stem cells and cell-mediated calcium deposition ${ }^{13}$.

In this regard, carbon nanomaterials like graphene derivatives could be useful. Graphene-based nanoparticles crosslinks have been used to enhance the mechanical properties of Col ${ }^{14-16}$. For example, alginate-chitosan-Col based composite scaffolds consisting of Graphene oxide (GO) fabricated by chemical crosslinking method increased mechanical properties compared to non-crosslinked and without GO counterparts ${ }^{17}$. Graphene is the most popular carbon nanomaterials, which is one atomic layer of $\mathrm{sp}^{2}$ hybridized carbon atoms. $\mathrm{GO}$ is a graphene layer decorated with oxygen-containing functionalities that are reduced in reduced graphene oxide ( $\mathrm{rGO}$ ). Graphene shows large surface area $\left(\sim 2600 \mathrm{~m}^{2} \mathrm{~g}^{-1}\right)$, high thermal and electrical conductivity and significant mechanical properties, known as the thinnest, strongest, and stiffest material ever found ${ }^{1,18}$. The physical, chemical and mechanical properties of graphene derivatives make them ideal for bone regeneration research. Large surface area, 
biocompatibility, easy handling, tunable mechanical and electrical characteristics make graphene a promising substrate for different cell/stem cell culture and bone studies. Easily chemical modification of graphene surface allows cell adhesion and control over their proliferation and differentiation into osteogenic lineages ${ }^{118}$. Graphene provides mechanical stiffness, electrical conductivity and chemical modifications for bone scaffolds. Graphene derivatives (alone or composite with other materials) have been extensively studied for bone repair researches. A study on periodontal ligament stem cells culture on 2- and 3D graphene substrates showed enhanced osteogenic differentiation after 28 days. Mouse mesenchymal stem cells cultured on chitosan-Gelatin-Go scaffold promoted differentiation into osteoblast in vitro ${ }^{19}$. In vivo studies of the structure showed increased Col deposition and accelerated bridging in rat tibial bone defect. Wua et al, ${ }^{20}$ reported that osteoinductive properties of graphene enhance cell adhesion and proliferation in Poly(lactic-co-glycolic acid) (PLGA) films cultured with BMMSCs. Better in vivo guiding bone regeneration showed by graphene-PLGA defect implants than PLGA group. They claimed that graphene increased osteogenic differentiation and bone regeneration through $\mathrm{PI} 3 \mathrm{~K} / \mathrm{Akt} / \mathrm{GSK}-3 \beta / \beta$-catenin signal circuit. Graphene incorporation into various forms of Col have used for regeneration of different tissues like pegylated GO-Col hybrid scaffold for diabetic wound repair ${ }^{21}, 3 \mathrm{D}$ rGO-Col for neural differentiation ${ }^{22}$, GO-Alginate-Chitosan-Col for in vitro bone tissue engineering ${ }^{17}$ and Col functionalized with GO for rat cranial defect repair ${ }^{23}$.

In this study, we synthesized Col and rGO coated Col (rGO-Col) scaffolds by chemical crosslinking and freeze-drying methods. Surface topography, mechanical and chemical properties of rGO-Col scaffolds were characterized and the results were compared with that without coating. Furthermore, human bone marrow-derived mesenchymal stem cells (hBMSCs) adhesion and proliferation on scaffolds were studied. Finally, bone formation studies were performed by scaffolds implantation into rabbit cranial bone defects.

\section{Materials And Method}

\section{Scaffold fabrication}

Col and Col-Go scaffolds were synthesized via chemical crosslinking and freeze-drying methods according to our previously published protocols ${ }^{14,24}$. Col type I (CCS-1, NZA, Iran) solution (1\% acetic acid $\mathrm{v} / \mathrm{v}$ ) was used for Col scaffold synthesis. The Col solution was cast in Teflon molds and was kept at -20 ${ }^{\circ} \mathrm{C}$ for $5 \mathrm{~h}$ and at $-80{ }^{\circ} \mathrm{C}$ for $12 \mathrm{~h}$, respectively. Then, it was freeze-dried at $-50{ }^{\circ} \mathrm{C}$ for $48 \mathrm{~h}$ (freeze drier, ALPH1-2LD, UK). In the next step, crosslinking solution including 1-ethyl-3-(3-dimethylaminopropyl) carbodiimide (EDC; Sigma-Aldrich) and N,hydroxysuccinimide (NHS, Sigma-Aldrich) in ethanol ( $90 \% \mathrm{v} / \mathrm{v}$ ) was added into the samples followed by further freeze-drying for $24 \mathrm{~h}$.

Go solution $(400 \mu \mathrm{g} / \mathrm{mL})$ was used for the coating of Col scaffolds according to our previously published protocol ${ }^{14}$. Before the coating process, water/GO/EDC (1000:5:4 weight ratio, respectively) was prepared and stirred for $15 \mathrm{~min}$ to activate carboxyl group on the $\mathrm{GO}$ plate's surface. The coating process was performed by immersion of Col scaffolds in water/GO/EDC solution for $6 \mathrm{~h}$ at room temperature. Then, 
the scaffolds were rinsed with DI water and the final Col-Go scaffolds were obtained by freeze-drying for $24 \mathrm{~h}$. Preparing Col-rGO scaffolds group, sodium hydrosulfite solution $\left(\left(\mathrm{Na}_{2} \mathrm{~S}_{2} \mathrm{O}_{4}\right.\right.$, Sigma-Aldrich) $(2 \%, 3$ min) was used to reduce Go coating. The Col and rGO-Col scaffolds were used for characterizations, in vitro and in vivo studies.

\section{Scaffolds characterization}

Porous structure and morphology of the scaffolds were studied by field emission scanning electron microscopy (FESEM) (Tescan, Vega II, Czech). For FESEM studies, the scaffolds were dried and coated with gold. Raman spectroscopy (Excitation laser source of $532 \mathrm{~nm}$; Jesco Japan) (only for the rGO-Col group) and Fourier transform infrared (FT-IR) spectrophotometer (500 to $3500 \mathrm{~cm}^{-1}, 4.0 \mathrm{~cm}^{-1}$ resolution; Equinox-55, Bruker, Madison, Wisconsin, USA) were used for chemical studies of the scaffolds and rGO coating. Liquid displacement method was used to calculate the porosity of Col and Col-rGO scaffolds. Ethanol was used as a liquid medium, according to the following equation 25 :

Porosity $(\%)=\left(\mathrm{W}_{1}-\mathrm{W}_{2}-\mathrm{W}_{\mathrm{S}}\right) /\left(\mathrm{W}_{3}-\mathrm{W}_{2}\right) \times 100$

Where W3 is the weight of the ethanol dish; W1 is the ethanol dish's weight after submersion of each scaffold; W2 is the ethanol dish's weight after removing each scaffold; and $W_{S}$ is the weight of each dried scaffold. Mechanical properties of the samples were measured by compressive testing (Instron, Norwood, MA, USA). Prepared samples (height $\approx 7 \mathrm{~mm}$, diameter $\approx 9 \mathrm{~mm}$ ) were forced by a $1 \mathrm{~mm} / \mathrm{min}$ loading rate. Elastic modulus was calculated using stress-strain curves of compression data.

\section{Cell culture and cellular evaluations}

Cellular experiments were conducted using hBMSCs cells. In vitro cytotoxicity assays were performed in 3 different groups, Col, Col-rGO scaffolds and TCP. Stem cells were cultured in the standard culture medium included with Dulbecco's modified Eagle medium (DMEM, Invitrogen), fetal bovine serum (FBS, 10\% (v/v), Gibco) and antibiotic (1\% penicillin/streptomycin, Sigma-Aldrich). Cells were incubated in an incubator at $37{ }^{\circ} \mathrm{C}$ and $5 \% \mathrm{CO}_{2}$. Cell passage were performed after $80 \%$ confluency and passage 3 cells were used for cellular studies.

Before cell culture, the scaffolds were sterilized by ethanol (75\%) and UV exposure. Cell viability and proliferation were examined by MTT (3-[4,5-dimethylthiazol-2-yl]-2,5-diphenyltetrazolium bromide) colorimetric assay with hBMSCs culture after 48 and $96 \mathrm{~h}$. For this assay, $10^{4}$ cells were seeded on Col, Col-rGO scaffolds and tissue culture plate (TCP) in 96-well culture plates. The samples were incubated in incubator at $37^{\circ} \mathrm{C}$ and $5 \% \mathrm{CO}_{2}$. At each time point, the culture medium was removed, then, MTT solution ( $5 \mathrm{mg} / \mathrm{mL}$ in PBS) was added to each sample and incubated for $4 \mathrm{~h}$. Then, MTT solution was removed and $100 \mu \mathrm{L}$ dimethyl sulfoxide (DMSO; Sigma-Aldrich) was added to each well in order to dissolve the formazone precipitates. At each time point, four samples were used and TCP was considered as the control group. The optical absorbance was measured using microplate reader (ELISA reader, BioTek) at $570 \mathrm{~nm}$ wavelength. 
For morphology assessment, the samples were incubated with culture medium for $24 \mathrm{~h}$ in an incubator at $37^{\circ} \mathrm{C}$ and $5 \% \mathrm{CO}_{2}$. hBMSCs were cultured on the scaffolds for $48 \mathrm{~h}$. After that, scaffolds were rinsed by PBS and cells were fixed by $2.5 \%$ glutaraldehyde $(1 \mathrm{~h})$. Then, samples were dehydrated using ethanol series $(10,30,70,90$, and 100\%) and cell morphology was studied using FESEM microscopy.

\section{In vivo experiments}

\section{Craniofacial bone defect Surgery procedures}

Before animal surgery, the circular scaffolds ( $8 \mathrm{~mm}$ diameters, circular and $2 \mathrm{~mm}$ thickness) were prepared and sterilized by ethanol (75\%) and UV exposure. Four New Zealand white rabbits (average weight of 2-2.5 kg) were used for this experiment. Animals were treated according to the animal care guidelines (Access to food and water, $12 \mathrm{~h}$ lighting and $12 \mathrm{~h}$ darkness at 20-25 ${ }^{\circ} \mathrm{C}$ ) approved by the Ethics Committee of Aja University of Medical Sciences. The animals were anesthetized with a mixture of ketamine and xylazine intramuscular injection. The surgical site was shaved and scrubbed with povidone-iodine. After preparations, approximately $3 \mathrm{~cm}$ surgical midline incision was made and calvaria exposed after soft tissue and periosteum dissection. Then, two circular calvarial defects $(8 \mathrm{~mm}$ diameters) were created in each rabbit by surgical drill and under irrigation. The drilled bone was removed and each scaffold (Col and Col-rGO) was implanted in one defect. The tissue layers were closed by 3-0 absorbable sutures and the animals were watched for 12 weeks. All animal related methods are reported in accordance with ARRIVE guidelines.

\section{Histological study}

The animals were sacrificed 12 weeks after surgery. Specimens were prepared for Hematoxylin-Eosin (H\&E) and alizarin red staining assays. The bone specimen, including scaffold sections and additional surrounding host bone, were removed for analysis. Rabbit bones were decalcified in $10 \%$ EDTA solution (Sigma Aldrich, USA) followed by being fixed by paraffin and stained with H\&E and alizarin red. The sections were taken from different parts of the samples and observed by light microscopy (Leica Microsystems AG, Germany).

\section{Results And Discussion}

Different natural or synthetic materials have been used for scaffold fabrication in bone tissue engineering field ${ }^{9,26-29}$. Natural materials have shown more appropriate regeneration properties, mostly due to their similarity to mammalian cell ECM. In this regard, Col is the most prevalent protein in human tissues and extensively used for biomaterial research ${ }^{9}$. Col exists in bone ECM and forms bone cell and tissue structural support, combined with mineral materials like calcium phosphate crystals. Col exclusive properties, including availability, biocompatibility, biodegradability, high porosity, and low immunogenicity, have resulted in extensive bone tissue engineering application ${ }^{9}$. Col provides easy 
processing and combination with other materials. Col has been used as injecting hydrogels, films and membranes, sponges and micro/nanospheres for bone regeneration studies ${ }^{9}$.

The macroscopic structure of Col and Col-rGO are shown in Fig. 1A and B, respectively. Graphene coating has darkened the scaffolds color. The structure and stability of the scaffold were not changed after the coating process. Figure 2 depicts morphological and structural characteristics of scaffolds during the synthesis process, resulted from FESEM. The results showed 3D porous structures in both scaffolds. The 3D structure of the Col scaffold did not change after GO coating and reduction process and 3D porous framework could be seen (Figs. 1 and 2). Bone scaffolds should provide 3D porous structure mimicking in vivo like bone-forming environment. Col's structure and composition flexibility have resulted in different structure scaffolds, including 3D porous Col sponges and hydrogels. An optimized protocol based on chemical crosslinking and freeze-drying resulted in a 3D porous scaffold (Fig. 2) which is in accordance with our previous studies ${ }^{14,24}$ and Liu et al.'s study ${ }^{30}$.

Raman spectroscopy was used to detect the typical graphene peaks on Col-rGO scaffolds. Fig. 3 shows three main peaks, D (Range of $\sim 1300-1400 \mathrm{~cm}^{-1}$ ), G (Range of $\sim 1500-1600 \mathrm{~cm}^{-1}$ ) and 2D (Range of $\sim 2700-2900 \mathrm{~cm}^{-1}$ ) in Col-rGO scaffolds confirming successful coating of graphene on Col scaffolds. The $\mathrm{G} / 2 \mathrm{D}$ ratio was used for determining graphene layers number. The ratio showed the coating of multi-layer graphene-coated structures. FTIR spectra of Col and Col-rGO scaffolds are shown in Fig. 4. Col scaffold spectra resulted amide I, II, III, and amide A peaks at 1628, 1535, 1233 and $3274 \mathrm{~cm}-1$, respectively 24,31 . Col-rGO scaffolds spectra showed the same amide groups in addition to 1615 and $2929 \mathrm{~cm}^{-1}$ peaks related to CO-NH stretching peak ${ }^{14,32}$. Chemical crosslink bonds Col amine or hydroxyl groups and GO sheets oxygen-containing functional groups (Carboxyl group ( $\mathrm{COOH}$ ) and alkoxy) (Fig. 3 and 4).

The liquid displacement method showed $96.4 \%$ porosity for Col and $94 \%$ for Col-rGO scaffolds, respectively. The porosity difference was not significant between scaffolds before and after rGO coating showing fabrication of highly porous scaffolds for cellular studies. Compressive testing was used to determine the effects of graphene coating on the Col scaffold's mechanical properties. Stress-strain curves of compression test on Col and Col-rGO scaffolds was used to determine elastic modulus and mechanical strength. The obtained results showed $325 \pm 18 \mathrm{kPa}$ of Elastic modulus for Col-rGO scaffold compared to that of $115 \pm 16 \mathrm{kPa}$ for Col samples. Bone is a load-bearing tissue with high strength properties and in result bone tissue scaffolds need to have sufficient mechanical properties. It is shown that Col has weak mechanical properties for bone structural support and bone differentiation ${ }^{9,33}$, requiring an additional part to enhance scaffold strength. It reported that Col's mechanical properties could be enhanced by crosslink to graphene-based nanoparticles ${ }^{14-16}$. Liu et al. ${ }^{30}$ significantly increased the elastic modulus of Col from $0.2 \mathrm{MPa}$ to $0.34 \mathrm{MPa}$ adding $0.1 \% \mathrm{GO}$. In this study, Col and Col-rGO scaffolds represented $115 \pm 16 \mathrm{kPa}$ and $325 \pm 18 \mathrm{kPa}$ elastic modulus, respectively, similar to our previous study ${ }^{14}$. Graphene nanosheets coating on Col structure remarkably enhanced mechanical strength of the scaffold by 2.8 folds. Using this scaffold, cells use mechanosensing and differentiate on a substrate similar to native bone tissue. In addition, implantation of a high-strength scaffold prevents 
additional scaffold and bone remodeling and damage to the injured area. Besides, the limited electrical conductivity of natural biomaterials challenges their practical applications. Its reported that osteoinductive properties of graphene incorporated PLGA films enhance bone differentiation of stem cells and guiding bone tissue regeneration ${ }^{20}$. Therefore, exceptional mechanical and electrical conductivity of rGO which is significantly more electrical conductive than GO on Col scaffolds seems to be helpful in bone repair.

Cytotoxicity of the samples was evaluated using MTT assay. After cell culture, MTT results (Fig. 5) showed more biocompatibility in Col-rGO scaffolds than alone Col, which was significant after $96 \mathrm{~h}(\mathrm{p}>$ 0.05). These results confirmed that addition of graphene not only did not induce any cytotoxic effects but also enhanced cell viability and proliferation. The adhesion of BMSCs on scaffolds was evaluated by FESEM microscopy. After $48 \mathrm{~h}$ of hBMSCs seeding on scaffolds, images were taken that are shown in Fig. 6. Stem cells were adhered and grown on both scaffolds. In both groups, the stem cells were attached and expanded on 3D scaffolds and penetrated into pores. In Col-rGO scaffolds, 3D adherence and expansion with natural morphology are apparent. Cells used the 3D porous structure of graphenecoated scaffolds for enhanced adhesion, proliferation and cell-cell contact.

Go and rGO cytotoxicity depends on the number of layers, concentration, lateral dimensions, shapes, and cell types ${ }^{12,24}$. For example, our previous study on different concentration ranges of $\mathrm{rGO}$ from 200-800 $\mu \mathrm{g} / \mathrm{mL}$ on Col scaffold showed that $400 \mu \mathrm{g} / \mathrm{ml}$ concentration allows highest cell viability for HUVECs cells ${ }^{14}$. Another study reported that $0.1 \% \mathrm{GO}$-Col aerogels group shows better cytocompatibility than $0.05 \%$ and $0.2 \%$ groups on rat BMSCs ${ }^{30}$. The addition of GO to alginate-chitosan-Col based composite scaffolds did not change the metabolic activity of MC3T3 osteoblast cells. It provided better support for cell proliferation compared to the GO nonincorporated group ${ }^{17}$. GO addition induced increased osteogenesis and in result, calcium mineral deposition. Cytotoxicity results of this study on Col-rGO showed more biocompatibility than alone Col on hBMSCs after $96 \mathrm{~h}$ seeding (Fig. 5). The same results were achieved about cell adhesion (Fig. 6). Despite adhesion and expansion on both scaffolds, Col-rGO scaffolds showed enhanced adhesion, proliferation, expansion, called cells developing spreading cytoplasmic projections, penetration into pores and cell-cell contact.

Different structural and physicochemical characteristics of Col-rGO scaffolds could be involved in these cellular results. The 3D structure of the Col-rGO scaffold provides high surface area for hBMSCs stem cells to attach and grow in an in vivo like structure and in different directions. Tissue scaffold should have 3D structure and interconnected pores for appropriate cell adhesion and interconnection, in addition to nutrients and oxygen transport. Significant porosity of $94 \%$ for Col-rGO scaffold (higher than $83 \%$ for the GO-Col scaffold synthesized by mixing of $\mathrm{Col}$ and $\mathrm{GO}$ solutions ${ }^{30}$ ) is sufficient for cell migration and vascularization ${ }^{34,35}$. High porosity facilitates stem cells interconnection and medium transport. Creating of cellular framework could be seen in Col-rGO scaffolds which is necessary for constructing larger tissue. Besides, the scaffolds' topological properties provide additional support for better cell adhesion, proliferation, and viability. It is confirmed that ripples and wrinkles on graphene nanosheets result better 
cell adhesion 25,36,37. Stiffer scaffolds activate molecules involved in cell adhesion and proliferation ${ }^{30,38}$. It's been reported that high strength, 3D structure and porous graphene foam induced spontaneous osteogenic differentiation for $\mathrm{hMSCs}^{39}$. Other surface properties like existing oxygen-containing functional groups such as hydroxyl, carboxyl and epoxy and free surface $\pi$ electrons allow hydrogen bonding, $\pi-\pi$ interactions, and other surface reactions, providing additional surface reaction sites ${ }^{24,40}$. Kolanthai et al. ${ }^{17}$ reported negatively charged surface of GO is favored by osteoblast cell adhesion, growth and proliferation. Surface sites can adsorb serum proteins, such as fibronectin, in culture media and provide a hydrophilic surface, enhancing stem cell adherence, viability, and proliferation. These interactions are confirmed in other studies using different graphene materials $24,37,41,42$. The abovementioned properties encounter some reported graphene cytotoxicity damages like ROS production 43,44 , physical damage to the cell membrane by graphene sharp edges 45,46 .

Histological analysis was used to examine the presence of new bone formation in the defect area. $\mathrm{H} \& \mathrm{E}$ (Fig. 7) and alizarin red (Fig. 8) staining were performed on rabbit cranial defect samples. H\&E results showed no necrosis or inflammation in both groups. Above mentioned advantages of Col-rGO scaffolds could be involved in animal study results. Testing this theory, an $8 \mathrm{~mm}$ critical-sized rabbit cranial defect model was used. As shown in Fig. 7, H\&E results showed that new osteogenesis and cell migration in different regions of the implanted Col-rGO scaffolds are more than Col counterparts. Alizarin red staining was used to determine mineralization and bone formation. Alizarin red results (Fig. 8) showed that compared to the Col scaffolds, mineralization in central regions of the defect could be seen for implanted Col-rGO scaffolds. Similarly, the surrounding area of the defects shows more mineralization and new bone formation.

Enhanced in vivo results follows the biological, physicochemical and topological properties of the ColrGO scaffolds like 3D, porous structure, biocompatibility, enhanced cell adhesion, high mechanical strength and specific surface factors, which are discussed above. Obtained results are in accordance with similar in vivo studies. For example, $0.1 \% \mathrm{GO}$-Col aerogel implant in rat cranial defect models showed better bone repair than Col aerogel ${ }^{30}$. In vivo results of graphene-contained scaffolds indicate the scaffold's osteogenesis properties without any external ingredients like growth factors or cells. Different studies have been used external osteogenesis factors along with scaffold. For example, ${ }^{47}$ used ECM components, cells and growth factors along with Col scaffold for osteogenesis. Besides the discussed reasons, another two advantages of Col-rGO scaffolds for better in vivo bone-forming results could be angiogenic and antibacterial properties of graphene. Addition of $\mathrm{rGO}$ flakes within MSC spheroids upregulated the expression of VEGF growth factors, cell-ECM interaction and allowed cell signaling cascades ${ }^{42}$. Low levels of ROS production by GO incorporation and activation of phospho-eNOS and phospho-Akt by rGO could be pro-angiogenic signaling factors inducing vascularization $42,48,49$. Govindarajan et al. investigated that 3D porous Col aerogel shows cytocompatibility and wound healing and angiogenesis effects ${ }^{50}$. Our scaffold fabricated by Col-rGO400 $\mu \mathrm{g} / \mathrm{ml}$ confirmed VEGF induced angiogenesis after subcutaneous implant for 4 weeks ${ }^{24}$. In addition, graphene materials present 
antibacterial and antifungal properties ${ }^{51,52}$, promising for bone tissue engineering. Our previous study confirmed that the Col-rGO scaffold induces antibacterial effects against E. coli, S. aureus and S. pathogens through the loss of bacterial membrane integrity and generation of oxidative stress ${ }^{14}$. ColrGO scaffold's antibacterial properties could be advantageous in preventing biofilm and implant infection and subsequent successful practical applications.

\section{Conclusion}

In this study, we fabricated Col and Col-rGO scaffolds by chemical crosslinking and freeze-drying method. Our finding showed that Col-rGO scaffolds exhibited 3D porous structures with enhanced biochemical properties and mechanical strength than Col scaffolds. Moreover, Col-rGO scaffolds provided better adhesion, viability and proliferation for hBMSCs cells. For in vivo study in rabbit cranial defect models, Col-rGO scaffolds exhibited more bone formation than Col scaffolds. Altogether, the study showed that rGO coating improves Col scaffold properties and could be a promising implant for bone injuries.

\section{Declarations}

\section{Author contributions}

S.B. performed the experiments under the supervision of N.B. and M.S. S.B., of N.B. and M.S. analyzed the results. The manuscript was written by S.B. and was finalized with all authors's assistance. All authors read and edited the manuscript.

\section{Competing interests}

The authors declare no competing interests.

\section{References}

1. Holt, B. D., Wright, Z. M., Arnold, A. M. \& Sydlik, S. A. Graphene oxide as a scaffold for bone regeneration. Wiley Interdisciplinary Reviews: Nanomedicine and Nanobiotechnology 9, e1437 (2017).

2. Walmsley, G. G. et al. Nanotechnology in bone tissue engineering. Nanomedicine: Nanotechnology, Biology and Medicine 11, 1253-1263 (2015).

3. Mathur, A., Ma, Z., Loskill, P., Jeeawoody, S. \& Healy, K. E. In vitro cardiac tissue models: Current status and future prospects. Advanced drug delivery reviews (2015).

4. Loh, Q. L. \& Choong, C. Three-dimensional scaffolds for tissue engineering applications: role of porosity and pore size. Tissue Engineering Part B: Reviews 19, 485-502 (2013).

5. Nie, W. et al. Three-dimensional porous scaffold by self-assembly of reduced graphene oxide and nano-hydroxyapatite composites for bone tissue engineering. Carbon 116, 325-337 (2017). 
6. Zhang, D., Wu, X., Chen, J. \& Lin, K. The development of collagen based composite scaffolds for bone regeneration. Bioactive materials 3, 129-138 (2018).

7. Baheiraei, N., Jalise, S. Z. \& Sanei, S. A. Recent advances in bioglass applications for bone tissue engineering. Pathobiology Research 20, 1-22 (2017).

8. Liu, X. \& Wang, S. Three-dimensional nano-biointerface as a new platform for guiding cell fate. Chemical Society Reviews 43, 2385-2401 (2014).

9. Ferreira, A. M., Gentile, P., Chiono, V. \& Ciardelli, G. Collagen for bone tissue regeneration. Acta biomaterialia 8, 3191-3200 (2012).

10. Sheyn, D. et al. Human induced pluripotent stem cells differentiate into functional mesenchymal stem cells and repair bone defects. Stem cells translational medicine 5, 1447-1460 (2016).

11. Arakawa, C. et al. Photopolymerizable chitosan-collagen hydrogels for bone tissue engineering. Journal of tissue engineering and regenerative medicine 11, 164-174 (2017).

12. Baheiraei, N. et al. Development of a bioactive porous collagen/ $\beta$-tricalcium phosphate bone graft assisting rapid vascularization for bone tissue engineering applications. Journal of Biomedical Materials Research Part A 106, 73-85 (2018).

13. Kajave, N. S., Schmitt, T., Nguyen, T.-U., Gaharwar, A. K. \& Kishore, V. Bioglass incorporated methacrylated collagen bioactive ink for 3D printing of bone tissue. Biomedical Materials (2020).

14. Norahan, M. H. et al. Electroactive cardiac patch containing reduced graphene oxide with potential antibacterial properties. Materials Science and Engineering: C 104, 109921 (2019).

15. Gao, C., Feng, P., Peng, S. \& Shuai, C. Carbon nanotube, graphene and boron nitride nanotube reinforced bioactive ceramics for bone repair. Acta biomaterialia 61, 1-20 (2017).

16. Yoon, O. J., Sohn, I. Y., Kim, D. J. \& Lee, N.-E. Enhancement of thermomechanical properties of poly (D, L-lactic-co-glycolic acid) and graphene oxide composite films for scaffolds. Macromolecular Research 20, 789-794 (2012).

17. Kolanthai, E. et al. Graphene oxide-a tool for the preparation of chemically crosslinking free alginate-chitosan-collagen scaffolds for bone tissue engineering. ACS applied materials \& interfaces 10, 12441-12452 (2018).

18. Dubey, N. et al. Graphene: a versatile carbon-based material for bone tissue engineering. Stem cells international 2015 (2015).

19. Saravanan, S. et al. Scaffolds containing chitosan, gelatin and graphene oxide for bone tissue regeneration in vitro and in vivo. International journal of biological macromolecules 104, 1975-1985 (2017).

20. Wu, X. et al. Enhanced osteogenic differentiation and bone regeneration of poly (lactic-co-glycolic acid) by graphene via activation of $\mathrm{PI} 3 \mathrm{~K} / \mathrm{Akt} / \mathrm{GSK}-3 \beta / \beta$-catenin signal circuit. Biomaterials science 6 , 1147-1158 (2018).

21. Chu, J. et al. PEGylated graphene oxide-mediated quercetin-modified collagen hybrid scaffold for enhancement of MSCs differentiation potential and diabetic wound healing. Nanoscale 10, 9547- 
9560 (2018).

22. Guo, W. et al. Construction of a 3D rGO-collagen hybrid scaffold for enhancement of the neural differentiation of mesenchymal stem cells. Nanoscale 8, 1897-1904 (2016).

23. Zhou, C. et al. Collagen functionalized with graphene oxide enhanced biomimetic mineralization and in situ bone defect repair. ACS applied materials \& interfaces 10, 44080-44091 (2018).

24. Norahan, M. H., Amroon, M., Ghahremanzadeh, R., Mahmoodi, M. \& Baheiraei, N. Electroactive graphene oxide-incorporated collagen assisting vascularization for cardiac tissue engineering. Journal of Biomedical Materials Research Part A 107, 204-219 (2019).

25. Bahrami, S. et al. Three-dimensional graphene foam as a conductive scaffold for cardiac tissue engineering. Journal of biomaterials applications 34, 74-85 (2019).

26. Najafloo, R., Baheiraei, N. \& Imani, R. Synthesis and characterization of collagen/calcium phosphate scaffolds incorporating antibacterial agent for bone tissue engineering application. Journal of Bioactive and Compatible Polymers, 0883911520966692 (2020).

27. Tebyanian, H. et al. Effects of collagen/ $\beta$-tricalcium phosphate bone graft to regenerate bone in critically sized rabbit calvarial defects. Journal of applied biomaterials \& functional materials 17 , 2280800018820490 (2019).

28. Shahrezaee, M. et al. Synthesis of magnesium doped amorphous calcium phosphate as a bioceramic for biomedical application: In vitro study. Silicon 10, 1171-1179 (2018).

29. Shahrezaie, M. et al. Effectiveness of tissue engineered three-dimensional bioactive graft on bone healing and regeneration: an in vivo study with significant clinical value. Journal of tissue engineering and regenerative medicine 12, 936-960 (2018).

30. Liu, S. et al. Biocompatible graphene oxide-collagen composite aerogel for enhanced stiffness and in situ bone regeneration. Materials Science and Engineering: C 105, 110137 (2019).

31. Deepachitra, R., Ramnath, V. \& Sastry, T. P. Graphene oxide incorporated collagen-fibrin biofilm as a wound dressing material. RSC Advances 4, 62717-62727, doi:10.1039/C4RA10150B (2014).

32. Liu, P., Huang, Y. \& Wang, L. A facile synthesis of reduced graphene oxide with Zn powder under acidic condition. Materials Letters $91,125-128$ (2013).

33. Zheng, Z. et al. Alignment of collagen fiber in knitted silk scaffold for functional massive rotator cuff repair. Acta biomaterialia 51, 317-329 (2017).

34. Venugopal, J. R. et al. Biomaterial strategies for alleviation of myocardial infarction. Journal of the Royal Society Interface 9, 1-19 (2012).

35. Norahan, M. H., Amroon, M., Ghahremanzadeh, R., Mahmoodi, M. \& Baheiraei, N. Electroactive graphene oxide-incorporated collagen assisting vascularization for cardiac tissue engineering. Journal of Biomedical Materials Research Part A (2018).

36. Xie, H., Cao, T., Gomes, J. V., Neto, A. H. C. \& Rosa, V. Two and three-dimensional graphene substrates to magnify osteogenic differentiation of periodontal ligament stem cells. Carbon 93, 266-275 (2015). 
37. Li, N. et al. Three-dimensional graphene foam as a biocompatible and conductive scaffold for neural stem cells. Scientific reports 3, 1-6 (2013).

38. Kang, S. et al. Covalent conjugation of mechanically stiff graphene oxide flakes to three-dimensional collagen scaffolds for osteogenic differentiation of human mesenchymal stem cells. Carbon $\mathbf{8 3}, \mathbf{1 6 2 -}$ 172 (2015).

39. Crowder, S. W. et al. Three-dimensional graphene foams promote osteogenic differentiation of human mesenchymal stem cells. Nanoscale 5, 4171-4176 (2013).

40. Goenka, S., Sant, V. \& Sant, S. Graphene-based nanomaterials for drug delivery and tissue engineering. Journal of Controlled Release 173, 75-88 (2014).

41. Park, J. et al. Graphene oxide flakes as a cellular adhesive: prevention of reactive oxygen species mediated death of implanted cells for cardiac repair. ACS nano 9, 4987-4999 (2015).

42. Park, J. et al. Graphene potentiates the myocardial repair efficacy of mesenchymal stem cells by stimulating the expression of angiogenic growth factors and gap junction protein. Advanced Functional Materials 25, 2590-2600 (2015).

43. Kanayama, I. et al. Comparative study of bioactivity of collagen scaffolds coated with graphene oxide and reduced graphene oxide. International journal of nanomedicine 9, 3363 (2014).

44. Chang, Y. et al. In vitro toxicity evaluation of graphene oxide on A549 cells. Toxicology letters 200, 201-210 (2011).

45. Hu, W. et al. Graphene-based antibacterial paper. ACS nano 4, 4317-4323 (2010).

46. Xie, C. et al. Elucidating the origin of the surface functionalization-dependent bacterial toxicity of graphene nanomaterials: Oxidative damage, physical disruption, and cell autolysis. Science of The Total Environment 747, 141546 (2020).

47. Liu, S. et al. Off-the-shelf biomimetic graphene oxide-collagen hybrid scaffolds wrapped with osteoinductive extracellular matrix for the repair of cranial defects in rats. ACS applied materials \& interfaces 10, 42948-42958 (2018).

48. Mukherjee, S. et al. Graphene oxides show angiogenic properties. Advanced healthcare materials 4, 1722-1732 (2015).

49. Xue, D. et al. Immunomodulatory properties of graphene oxide for osteogenesis and angiogenesis. International journal of nanomedicine 13, 5799 (2018).

50. Govindarajan, D. et al. Fabrication of hybrid collagen aerogels reinforced with wheat grass bioactives as instructive scaffolds for collagen turnover and angiogenesis for wound healing applications. ACS applied materials \& interfaces $9,16939-16950$ (2017).

51. Karahan, H. E. et al. Graphene materials in antimicrobial nanomedicine: current status and future perspectives. Advanced healthcare materials 7, 1701406 (2018).

52. Kumar, P., Huo, P., Zhang, R. \& Liu, B. Antibacterial properties of graphene-based nanomaterials. Nanomaterials 9, 737 (2019). 
Figures

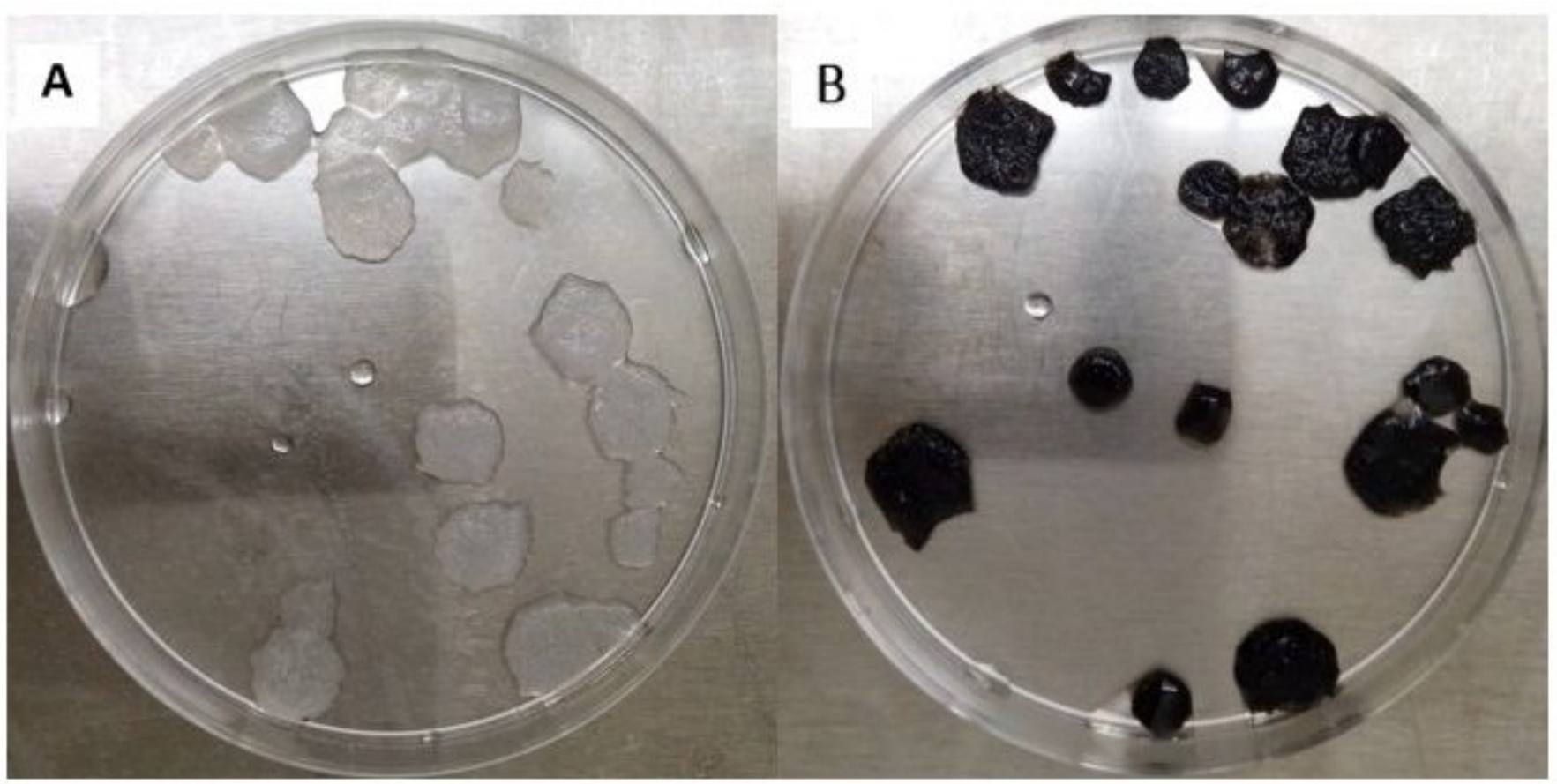

Figure 1

Macroscopic structure (A) Col and (B) Col-rGO scaffolds. 

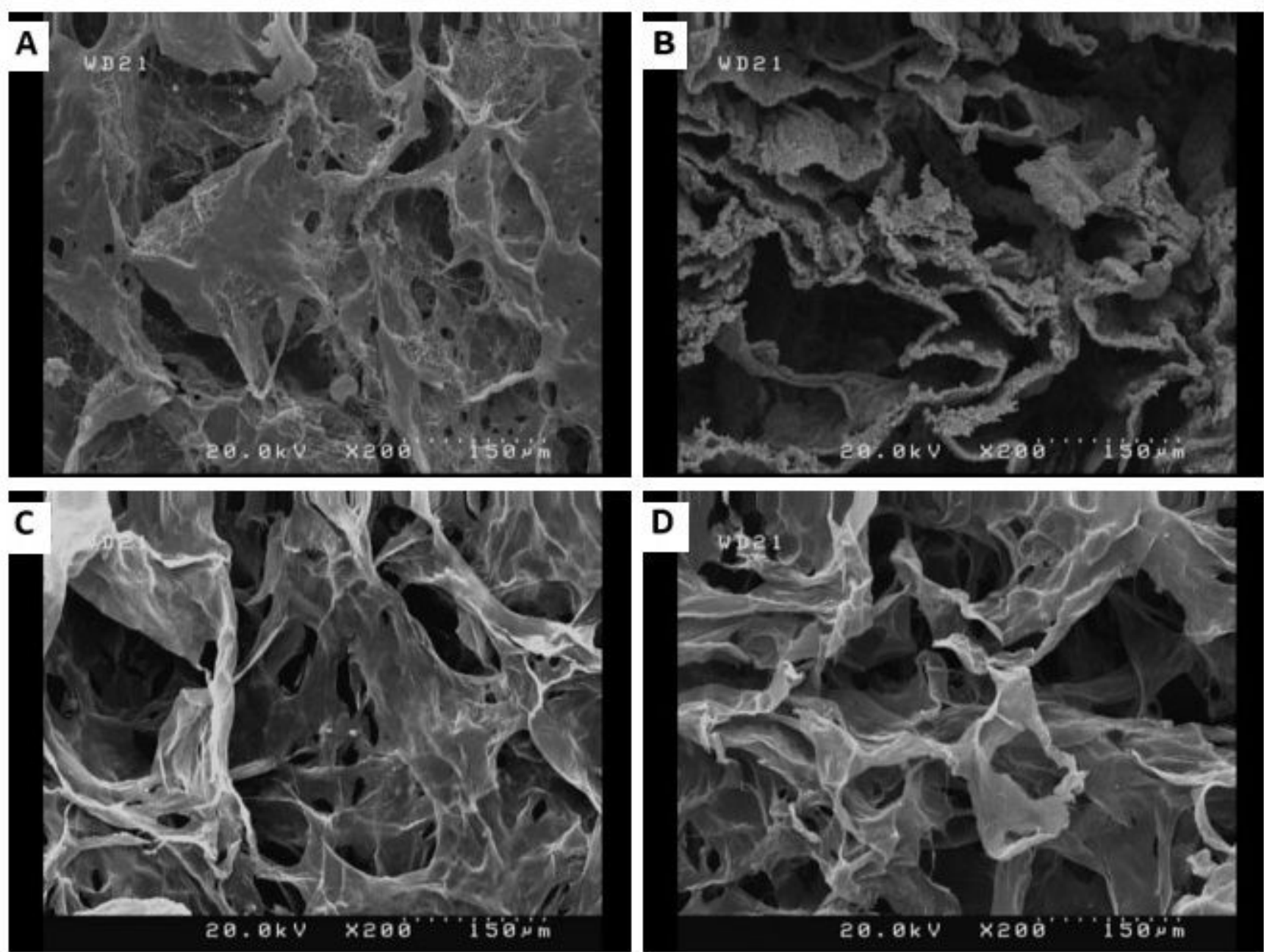

Figure 2

FESEM images of Col (A and B) before graphene coating and Col-rGO (C and D) scaffolds, depicting 3D porous scaffolds.

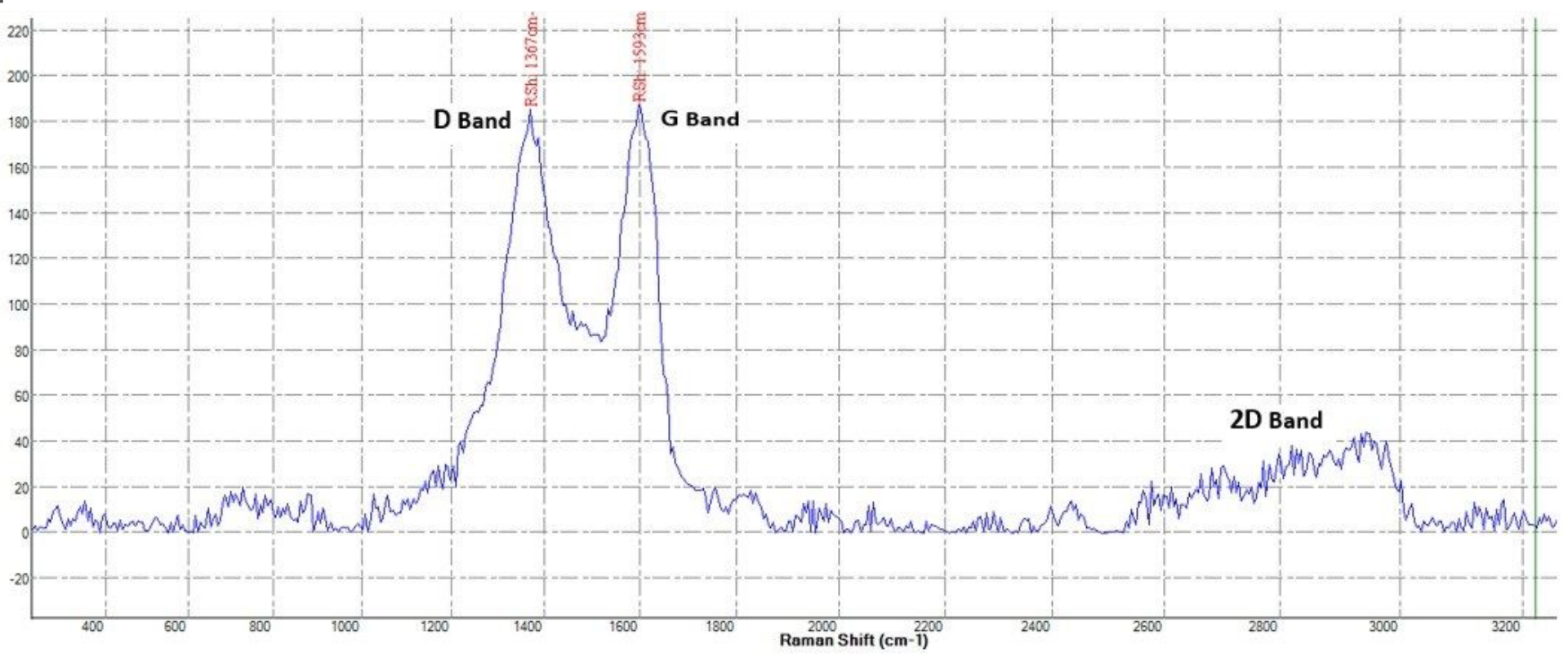

Figure 3 
Raman spectrum of the Col-rGO scaffolds showing D, G and 2D bands.

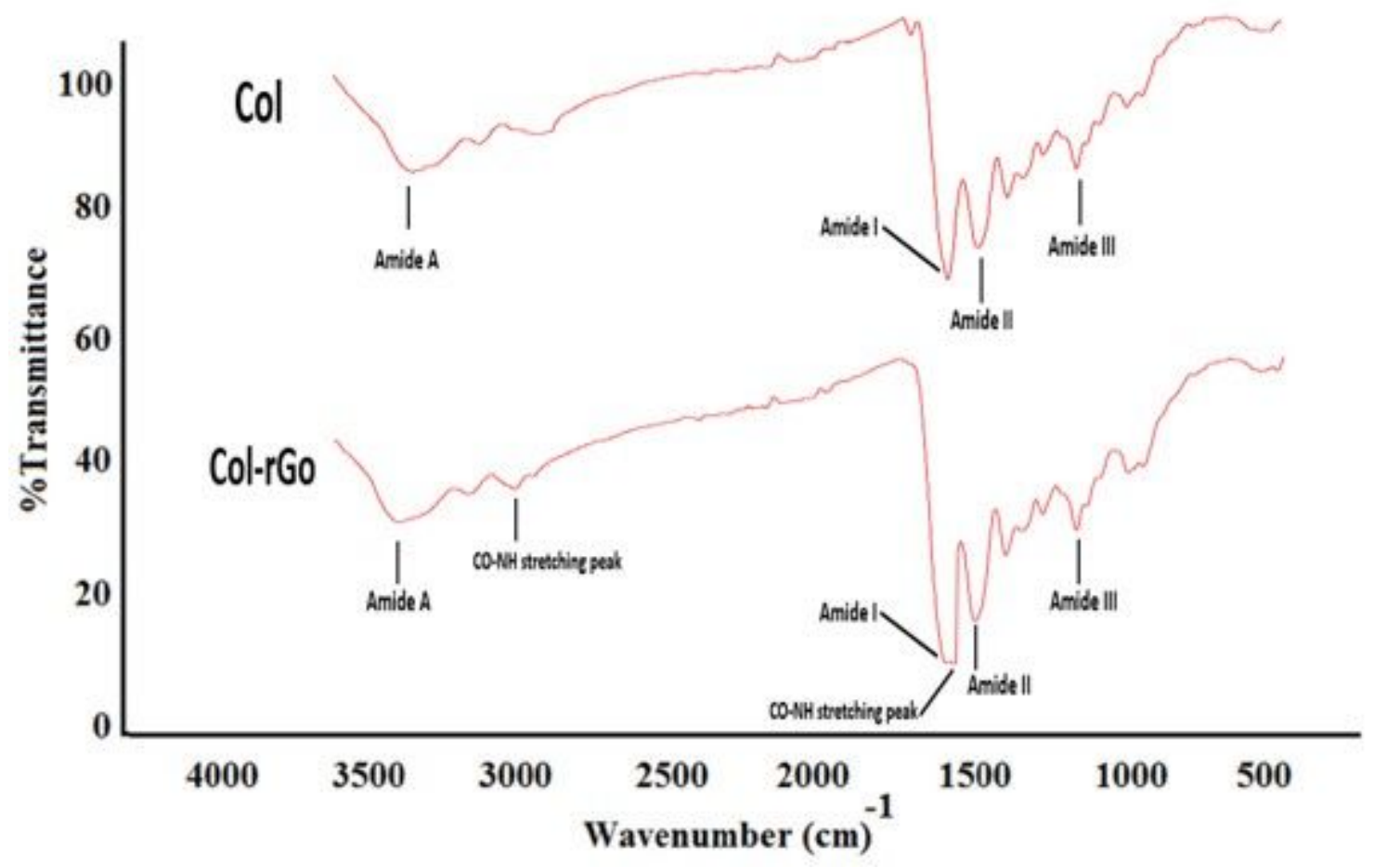

Figure 4

FTIR spectra of the Col and Col-rGO scaffolds.
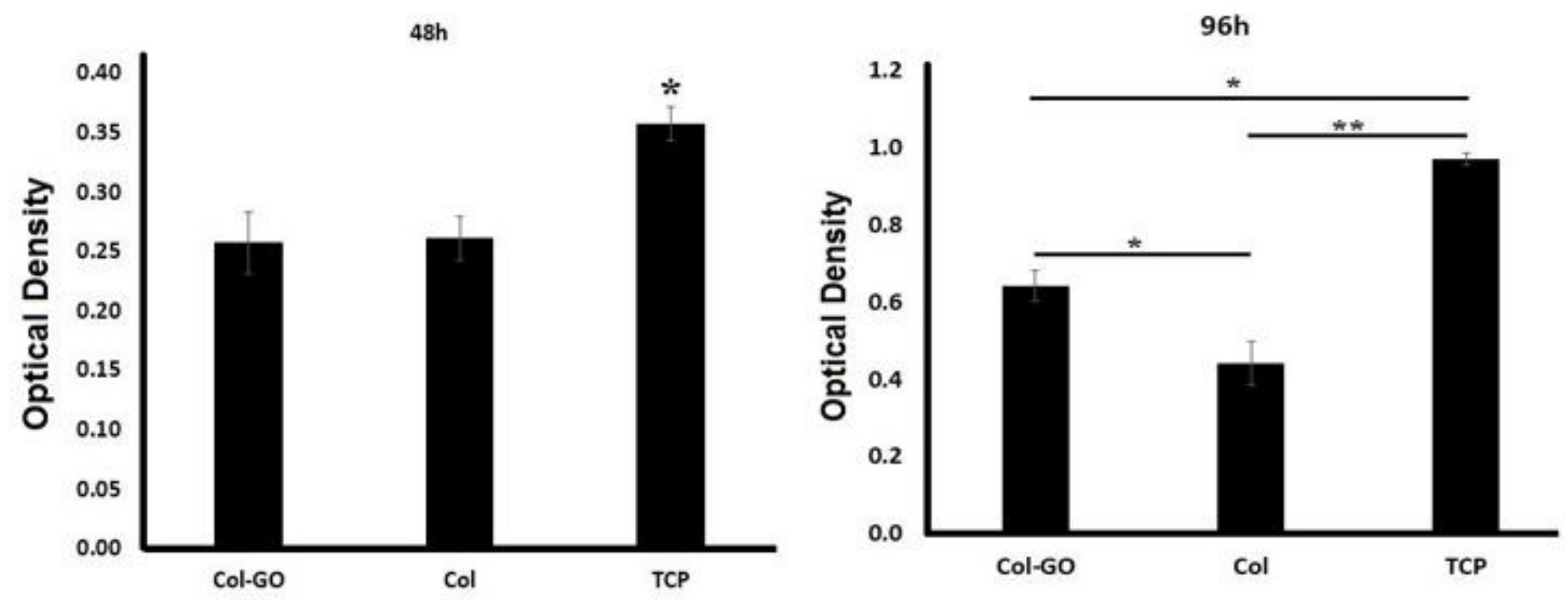

Figure 5

MTT assay results of hBMSCs culture on Col, Col-rGO scaffolds and TCP groups. The addition of graphene did not induce any cytotoxic effect. 

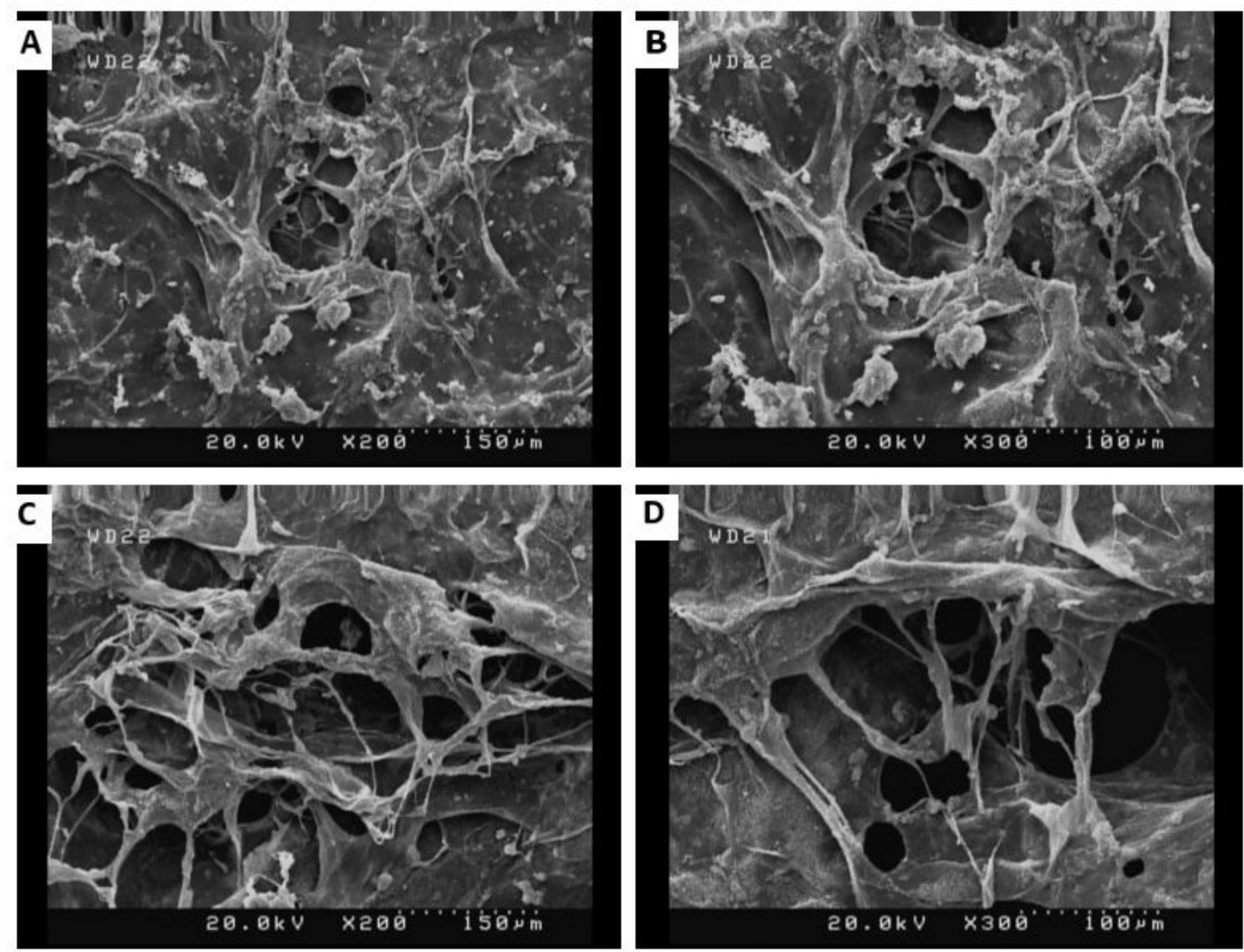

\section{Figure 6}

SEM images of hBMSCs seeded on Col (A and B) and Col-rGO (C and D) scaffolds after $48 \mathrm{~h}$. 

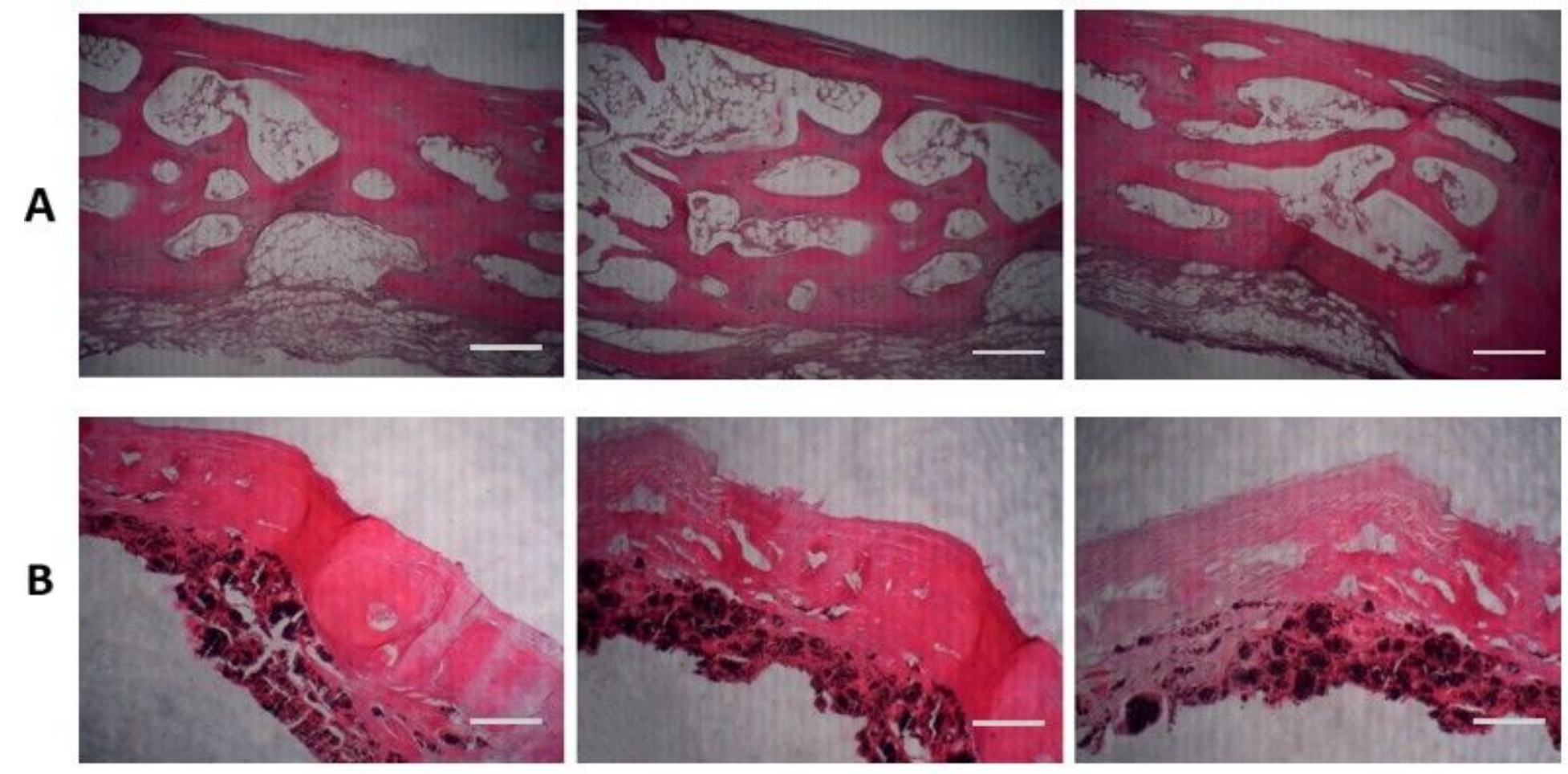

\section{Figure 7}

$\mathrm{H} \& \mathrm{E}$ staining of the bone defects area repaired by Col (A) and Col-rGO (B) scaffolds, 12 weeks postimplantation. Scale bar : $10 \mu \mathrm{m}$.
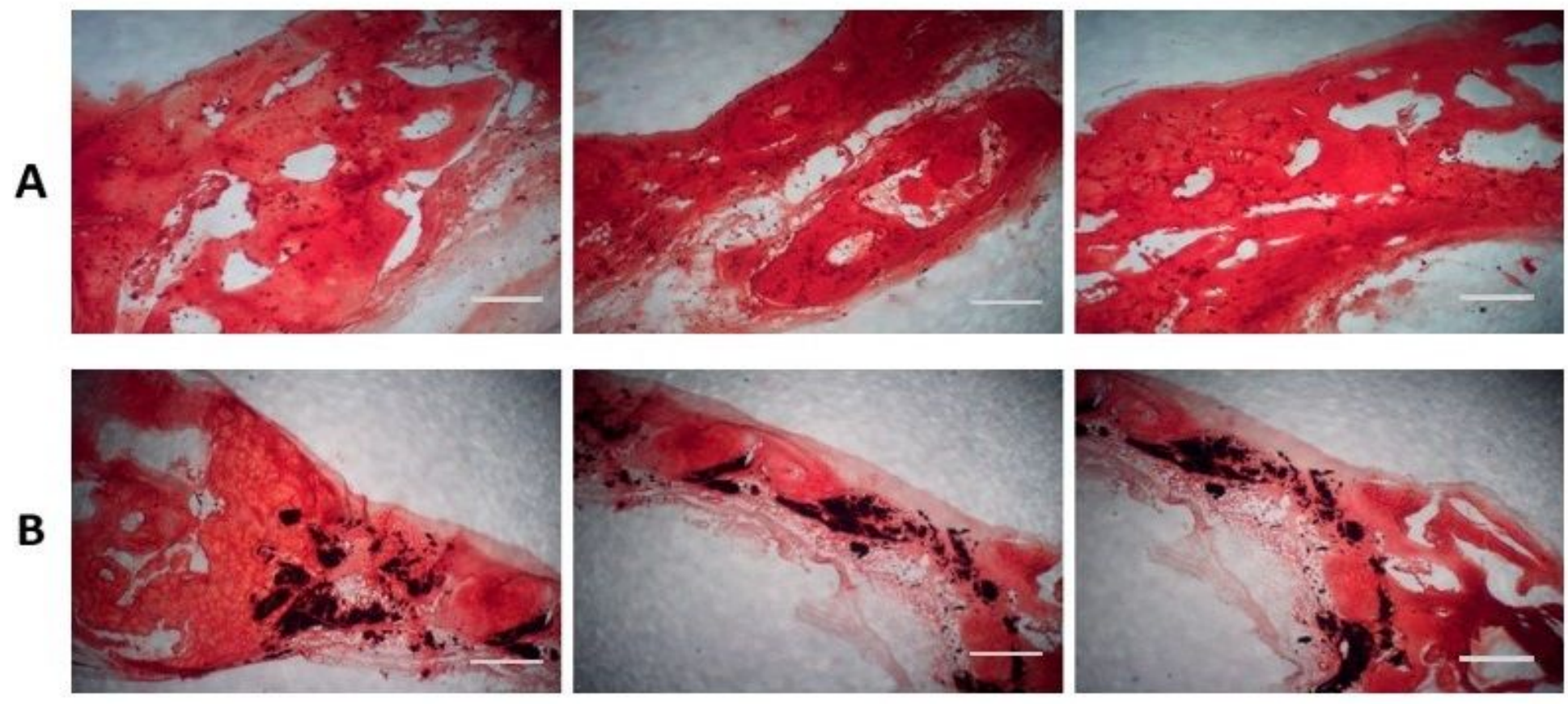

\section{Figure 8}

Alizarin red staining of the bone defects area repaired by Col (A) and Col-rGO (B) scaffolds, 12 weeks post-implantation. Scale bar : $10 \mu \mathrm{m}$. 